\title{
Distributed cortical structural properties contribute to motor cortical excitability and inhibition
}

\author{
Eran Dayan ${ }^{1,2,5^{*}}$, Virginia López-Alonso ${ }^{2,3,5}$, Sook-Lei Liew ${ }^{3}$, Leonardo G. Cohen ${ }^{1 *}$
}

1. Department of Radiology, Biomedical Research Imaging Center and Neuroscience Curriculum, University of North Carolina at Chapel Hill, Chapel Hill, NC

2. Human Cortical Physiology and Neurorehabilitation Section, National Institute of Neurological Disorders and Stroke, National Institutes of Health, Bethesda, MD

3. Faculty of Sciences of Sport and Physical Education, Department of Physical Education, University of A Coruña, A Coruña, Spain

4. Division of Occupational Science \& Occupational Therapy, Division of Physical Therapy \& Biokinesiology, Ostrow School of Dentistry; Department of Neurology, Keck School of Medicine; Stevens Neuroimaging and Informatics Institute, University of Southern California

5. These authors contributed equally to this work

* Correspondence: correspondence should be addressed to:

eran dayan@med.unc.edu (E.D) or cohenl@ninds.nih.gov (L.G.C), 10 Center Drive, Bethesda, MD 20892

\section{Abstract}

The link between the local structure of the primary motor cortex and motor function has been well documented. However, motor function relies on a network of interconnected brain regions and the link between the structural properties characterizing these distributed brain networks and motor function remains poorly understood. Here, we examined whether distributed patterns of brain structure, extending beyond the primary motor cortex can help classify two forms of motor function: corticospinal excitability and intracortical inhibition. To this effect, we recorded high-resolution structural magnetic resonance imaging scans in 25 healthy volunteers. To measure corticospinal excitability and inhibition in the same volunteers we recorded motor evoked potentials (MEPs) 
elicited by single-pulse transcranial magnetic stimulation (TMS) and short-interval intracortical inhibition (SICl) in a separate session. Support vector machine (SVM) pattern classification was used to identify distributed multivoxel gray matter areas, which distinguished subjects who had lower and higher MEPs and SICls. We found that MEP and $\mathrm{SICl}$ classification could be predicted based on a widely distributed, largely nonoverlapping pattern of voxels in the frontal, parietal, temporal, occipital and cerebellar regions. Thus, structural properties distributed over the brain beyond the primary motor cortex relate to motor function.

\section{Introduction}

Variation in local brain structure has been shown to be linked to performance in a range of motor functions (Kanai and Rees, 2011). Structure-function links of this sort were demonstrated at both the microstructural and macrostructural scales. For example, variation in microstructural white matter integrity in the body of the corpus callosum, as assessed with diffusion MRI is associated with variation in performance of a bimanual coordination task (Johansen-Berg et al., 2007). Similarly, individual differences in the macrostructural gray matter properties of the presupplementary motor area are linked to subjects' ability to voluntarily select correct actions in the face of conflict (van Gaal et al., 2011). However, the link between the structural properties characterizing distributed brain networks and motor function remains incompletely understood.

In humans, transcranial magnetic stimulation (TMS) techniques have been vital in probing the physiological properties of the motor system (Dayan et al., 2013; Hallett, 2007; Rothwell, 1997). Two transcranial magnetic stimulation (TMS) protocols have been widely utilized as markers of motor corticospinal excitation and inhibition at rest. Motor evoked potentials (MEPs) elicited by single-pulse TMS over the primary motor cortex (M1) are a widely-used measure of instantaneous corticospinal excitability (Hallett, 2007; Rothwell, 1997; Rothwell et al., 1999). Similarly, short-interval intracortical inhibition (SICl), elicited by paired-pulse TMS over M1 is widely regarded as a measure of cortical inhibition (Kujirai et al., 1993; Rothwell et al., 2009). MEPs and 
SICls relate with the structure of the primary motor cortex (Conde et al., 2012). However, since motor function does not rely solely on one cortical region ( $\mathrm{He}$ et al., 2007; Matsumoto et al., 2006; Picard and Strick, 1996), we examined links between distributed structural properties of the cerebral cortex and MEPs and SICls, an issue that has not been reported in the literature. We reasoned that since clear links between the structure or function of single brain regions and variability in subjects' response to TMS were not identified to date, examining multivariate distributed substrates may provide an alternative approach. We thus evaluated whether differences in the magnitudes of MEPs and SICls could be classified from subjects' distributed wholebrain multi-voxel patterns of gray matter volume using whole brain machine learning pattern classification analysis.

\section{Methods}

Subjects: Data from 25 young, right-handed healthy volunteers (13/12 females/males; mean age 26.48 \pm 5.15 STD) were used for analysis. Handedness was established using the Edinburgh Handedness Inventory (Oldfield, 1971). All subjects had unremarkable physical and neurological history, no MRI contradictions, and did not use any psychoactive medication. Written informed consent was obtained from all subjects prior to their participation in the study and all procedures were approved by the Combined Neuroscience Institutional Review Board, National Institutes of Health. All procedures were in accordance with approved guidelines.

General procedure: All subjects underwent an imaging session and a stimulation session, which were administered separately (Fig 1A). The imaging session consisted of an anatomical scan (see details beneath). The stimulation sessions comprised single and paired-pulse TMS protocols, administered in an interleaved manner, where MEPs and SICls were recorded respectively (Fig 1B). During the stimulation sessions, subjects were seated in a comfortable chair with their eyes open and were asked to stay relaxed and to not engage in conversation during the course of stimulation.

EMG recordings: Electromyographic (EMG) traces were recorded via $\mathrm{Ag} / \mathrm{AgCl}$ surface 
recording electrodes ( $7 \mathrm{~mm} \times 4 \mathrm{~mm}$ recording area), placed over the right first dorsal interosseous (FDI) muscle. The active electrode was placed over the muscle belly and the reference electrode over the metacarpophalangeal joint of the index finger. Responses were acquired using a Neuropack MEB-2200 device (Nihon Kohden, Tokyo, Japan) through filters set at $10 \mathrm{~Hz}$ and $2 \mathrm{kHz}$ with a sampling rate of $5 \mathrm{kHz}$, amplified (Micro-1401, Cambridge Electronic Devices, Cambridge, UK), and then recorded using the Signal software (Cambridge Electronic Devices, Cambridge, UK).

TMS procedure: TMS was delivered through a figure-of-eight coil with an outer diameter of $70 \mathrm{~mm}$ (Magstim Co., Whitland, Dyfeld, UK) over the left motor cortex. The stimulators were triggered using the Signal software. The coil was held with the handle pointing backwards and laterally to evoke an anteriorly directed current in the brain (Sakai et al., 1997), and was optimally positioned to obtain MEPs in the FDI muscle. Using this configuration, single and paired pulses were delivered from a monophasic Magstim BiStim stimulator. We first localized the "motor hotspot" (defined as the point on the scalp at which single pulse TMS elicited MEPs of maximal amplitude from the right FDI). We then established each subject's resting motor threshold (RMT), which corresponds to the minimum stimulation intensity over the motor hotspot, eliciting an MEP in the relaxed FDI of no less than $50 \mu \mathrm{V}$ in 5 out of 10 trials.

Overall, the stimulation session included 40 stimulation trials, including $20 \mathrm{MEPs}$ (at $120 \% \mathrm{RMT}$ ) and $20 \mathrm{SICl}$ measures. MEPs and SICls were administered in an interleaved and randomized manner, with an inter-trial interval of $5 \mathrm{~s}$, varying by up to 10\%. SICls were recorded as described previously (Kujirai et al., 1993), whereby a subthreshold conditioning stimulus (CS) at $80 \%$ of RMT preceded a test stimulus (120\% $\mathrm{RMT})$ by $3 \mathrm{~ms}$.

--Figure 1 here-

Imaging Setup: Imaging data was acquired with a 3.0-T GE Signa HDx scanner equipped with an 8-channel coil. High-resolution $\left(1 \times 1 \times 1 \mathrm{~mm}^{3}\right)$ 3D magnetization prepared rapid gradient echo (MPRAGE) T1-weighted images were acquired (repetition 
time $=4.688 \mathrm{~ms}$, echo time $=1.916 \mathrm{~ms}$, slice thickness: $1.2 \mathrm{~mm}$, slice spacing $=1.2$ $\mathrm{mm}$, acquisition matrix $=224 \times 224 \mathrm{~mm}^{2}$, flip angle $=12^{\circ}, 124$ slices).

\section{Data Analysis:}

\section{TMS Data Analysis:}

Mean peak-to-peak amplitudes served as our primary outcome measure. Trial-to-trial variability in MEP amplitudes were additionally quantified using the coefficient of variation $(\mathrm{CV})$, calculated across all MEP trials as follows:

$$
C V=\frac{\sigma}{\mu}
$$

where $\sigma$ denotes standard deviation and $\mu$ denotes the mean. For the $\mathrm{SICl}$ analysis, the mean peak-to-peak amplitude of the conditioned MEP was expressed as a percentage of the mean peak-to-peak amplitude of the unconditioned MEP. Trial-to-trial variation in $\mathrm{SICl}$ amplitudes was quantified using the CV metric, as described above. Parametric statistical tests were used to analyze MEP and $\mathrm{SICl}$ measurements after confirming the normality of the distributions using Kolmogorov-Smirnov tests. All tests were performed in SPSS 19 (Chicago, IL). Significance was set at alpha=0.05.

\section{Imaging data analysis}

Image preprocessing: The VBM8 toolbox, part of Statistical Parametric Mapping 8, was used to preprocess subjects' anatomical scans. The images were first normalized to the Montreal Neurological Institute (MNI) standard space and segmented for gray matter, white-matter and cerebro-spinal fluid (CSF) using the default segmentation routines of VBM8 (Gaussians per class 2,2,2,3,4,2; Bias regularization 0.0001; Bias FWHM 60mm cutoff; Affine regularization ICBM space template; Warping regularization 4; Sampling distance 3) and DARTEL normalization. The images were then subjected to pattern classification analysis, with the objective of finding distributed patterns of graymatter volume that could classify group differences in corticospinal excitability and inhibition at rest. This approach follows a recent body of research where multivariate 
morphometric parameters can be used to differentiate healthy controls from, for instance, patients with Alzheimer's disease (Vemuri et al., 2008) or autism (Ecker et al., 2010b) based on machine learning pattern classification techniques (Bishop, 2006).

Pattern Classification: Pattern classification analysis was performed using the Pattern Recognition of Brain Image Data (PROBID) toolbox, on MATLAB 7. This analysis aimed to find patterns of gray matter volume that accurately classify group differences in MEPs and SICls, treating subjects' images as points in a high-dimensional space, corresponding to the number of voxels contained on each image (Dayan et al., 2014; Ecker et al., 2010b). Pattern classification analysis was used, rather than a massunivariate approach, as it may potentially allow to detect more subtle multivariate structural substrates that contribute to variation in MEPs and SICls.

The subjects were first split (using a median split) into groups, distinguishing between subjects for whom low and high mean MEP amplitudes and low and high SICls were recorded (thus the 25 subjects were split into two groups of 12 subjects each, leaving out the median). The median split procedure enabled labeling of the data (into two groups in each classification), a prerequisite for supervised pattern classification. Modulated and normalized preprocessed gray matter images (see description of preprocessing steps, above) were then subjected to kernel support vector machine (SVM) classification (Boser et al., 1992). This procedure is composed of two phases. First, in the training phase, a kernel SVM classifier is trained to distinguish between modulated and normalized anatomical images, labeled according to the results of the median split analysis, described above. In this phase, a hyperplane that separates the images in the training dataset according to their known labels was defined. Then, during the test phase, the performance of the classifier was tested with a leave-two-out crossvalidation procedure, whereby the test was administered $n$ times ( $n=$ number of subjects), leaving a pair of subjects out on each iteration.

In this analytical framework accuracy also denotes the average between the classification's sensitivity (proportion of subjects from class label I that were correctly 
classified as such) and specificity (proportion of subjects from class label II, who were correctly classified as such). As the input space used for classification was in voxel space, the weight vector normal to the hyperplane defined during training corresponds to the direction along which the images belonging to two groups differ the most. These inputs were then used to generate discrimination maps, which depicted a spatial map of the voxels that contributed the most to the discrimination among groups (Ecker et al., 2010b; Marquand et al., 2010; Mourão-Miranda et al., 2005). The maps depict voxels whose weights were at least $60 \%$ of the value of the voxel with the highest weight overall. This conservative threshold (Ecker et al., 2010a; Mourão-Miranda et al., 2005) allowed us to focus on the regions which most strongly discriminated among the groups. Discrimination maps were smoothed with a $3 \mathrm{~mm}$ Gaussian kernel and cluster thresholded (10 voxels) for illustration purposes and the results were visualized using MRIcron (http://www.mccauslandcenter.sc.edu/mricro/mricron/).

Significance estimates for the accuracy of each classification were derived using a permutation test consisting of 5000 iterations. In this test, the classification procedure was repeated 5000 times, wherein labels were randomly assigned to subjects. In each permutation the cross-validation procedure was repeated and the number of times the accuracy levels exceeded those obtained with the original labeled data were counted, where $p$ denotes the accumulated number of times divided by 5000 .

\section{Results}

Data from 25 young healthy volunteers were analyzed, testing the utility of using SVM classification of volumetric patterns of gray matter to predict group differences in corticospinal excitability and inhibition at rest, as measured with TMS-induced MEPS and SICs.

\section{Classification of MEP amplitudes}

Subjects displayed substantial interindividual differences in mean peak-to-peak MEP amplitudes (Fig. 2A), which were normally distributed in this sample (Kolmogorov- 
Smirnov $Z=0.162, p=0.091)$ Indeed, a median split of the data into two groups $(n=12$ each), of subjects who displayed low and high mean MEP amplitudes (henceforth, $\mathrm{MEP}_{\text {low }}$ and $\left.\mathrm{MEP}_{\text {high}}\right)$ resulted in significant differences between the groups $\left(\mathrm{t}_{22}=5.234, \mathrm{p}\right.$ $<0.0001$ ), confirming the existence of sizable interindividual differences. These two groups did not differ in age $\left(\mathrm{t}_{22}=0.922, \mathrm{p}=0.366\right)$ or in their male/female distributions (Kolmogorov-Smirnov $Z=1.021, P=0.249$ ).

We first sought to test the degree to which group differences in MEP amplitudes could be predicted using whole-brain SVM classification. $75 \%$ of the subjects in the MEP low group (the classification model's 'sensitivity') and $58.33 \%$ of the subjects in the $\mathrm{MEP}_{\text {high }}$ group (the model's 'specificity') were classified correctly, resulting in an overall accuracy of $66.67 \%$, which was significantly better than chance ( $p<0.05$, random permutation test) (Fig. 2B). Thus, these results reveal that patterns of gray matter allowed for a classification of group differences in MEP amplitudes. To more specifically identify the regions that contributed to this classification, discrimination maps were generated, depicting the weight of the voxels which contributed the most to the discrimination among the $\mathrm{MEP}_{\text {low }}$ and $\mathrm{MEP}_{\text {high }}$ groups (Fig. 2C, Table 1). The maps revealed that a widely distributed pattern of voxels composed of bilateral frontal and middle temporal, right inferior and anterior parietal and inferior occipital and left posterior cerebellar foci discriminated among the two groups.

--Figure 2 and Table 1 around here--

We next quantified the trial-to-trial variability in amplitudes with the CV statistic (Fig. 3A). This allowed us to assess the contribution of more transient and unspecific factors to the classification of MEPs (for instance, movement of the TMS coil along the stimulation site, slight changes in the orientation of stimulation, etc). MEP CVs differed substantially among subjects and were insignificantly correlated with mean MEP amplitudes ( $r=-$ $0.268, p=0.195$ ), suggesting that these two measures were largely independent of one another in the current sample of subjects. A median split of the data into two groups $\left(\mathrm{n}=12\right.$ each) of subjects who displayed high and low CVs (henceforth, MEPCV $\mathrm{V}_{\text {low }}$ and $\left.M E P C V_{\text {high }}\right)$ resulted in significant difference between the two groups $\left(t_{22}=5.53, p\right.$ 
$<0.0001)$. These two groups did not differ in age $\left(\mathrm{t}_{22}=0.269, \mathrm{p}=0.791\right)$ or in their male/female distribution, which were identical.

The trial-to-trial variation in MEP amplitudes could not be accurately classified in relation to gray matter volumetric patterns. Only $41.67 \%$ of the subjects in the MEPCV low (sensitivity) and $58.33 \%$ of the subjects in the $\mathrm{MEPCV}_{\text {high }}$ (specificity) groups were classified correctly, resulting in an overall accuracy of $50 \%$ (Fig. 3B). The accuracy of this classification, which did not differ from chance levels, was not statistically significant $(p=0.58)$

--Figure 3 here--

\section{Classification of SICl amplitudes}

$\mathrm{SICl}$ amplitudes were also variable among subjects (Fig. $4 \mathrm{~A}$ ) and the data was also normally distributed in this sample (Kolmogorov-Smirnov $Z=0.126, p=0.2$ ). Indeed, a median split of the data into two groups ( $n=12$ each) of subjects who displayed low and high mean SICls (henceforth, $\mathrm{SICl}_{\text {low }}$ and $\mathrm{SICl}_{\text {high }}$ ) resulted in significant differences between the groups $\left(t_{22}=6.48, p<0.0001\right)$. These two groups did not differ in age $\left(t_{22}=0.154, p=0.879\right.$ ) or in their male/female distributions (Kolmogorov-Smirnov $Z=0.408$, $\mathrm{P}=0.996)$.

We next tested whether $\mathrm{SICl}$ mean amplitudes could be predicted using whole brain SVM classification. $58.33 \%$ of the subjects in the $\mathrm{SICl}_{\text {low }}$ group (the model's sensitivity) and $75 \%$ of the subjects in the $\mathrm{SICl}_{\text {high }}$ group (the model's specificity) could be classified correctly (Fig 6A), which together summed up to $66.67 \%$ accuracy, significantly better than chance levels ( $p<0.05$, random permutation test; Fig 4B). Discrimination maps were then generated in order to identify the regions which contributed to this classification. A distributed pattern of voxels in bilateral frontal and posterior cerebellar and left inferior occipital and inferior parietal foci contributed mostly to the classification of group differences in $\mathrm{SICl}$ amplitudes (Fig 4C, Table 2).

--Figure 4 and Table 2 around here-- 
The trial-to-trial variability in SICls, quantified with the CV statistic (Fig. 5A), differed substantially among subjects and was significantly inversely correlated with mean SICls $(r=-0.498, p<0.02)$. A median split of this data into two groups $(n=12$ each) of subjects who displayed high and low SICI CVs (henceforth, SICICV significant difference between the two groups $\left(t_{22}=5.818, p<0.0001\right)$. These two groups did not differ in age $\left(\mathrm{t}_{22}=0.4, \mathrm{p}=0.969\right)$ or in their male/female distributions (KolmogorovSmirnov $Z=0.408, P=0.996)$.

We next assessed the degree to which group differences in the trial-to-trial variability of SICls could be predicted using whole brain SVM classification. $41.67 \%$ of the subjects in the $\mathrm{SICICV}_{\text {low }}$ group (sensitivity) and $33.33 \%$ of the subjects in the $\mathrm{SICICV}_{\text {high }}$ group (specificity) were classified correctly. Combined, whole brain SVM of SICI CVs thus resulted in $37.5 \%$ accurate classification, a proportion which was not statistically significant ( $p=0.925$; Fig. 5B).

--Figure 5 here-

\section{Similarities between MEP and SICl classification}

Finally, we assessed similarities in the classification of MEPs and SICls, as our results indicate that both were statically significant. In the current dataset MEPs and SICls were insignificantly correlated within subjects $(r=-0.169 . p=0.419)$. Overlaying the discrimination maps identified in the classification of each of these measures (Fig. 2C and Fig. $4 \mathrm{C}$ ) revealed that the maps were largely non-overlapping (Fig. 6).

--Figure 6 here--

\section{Discussion}

We tested the feasibility of classifying group differences in mean MEP and $\mathrm{SICl}$, elicited by single and paired-pulse stimulation of M1, from multi-voxel patterns of gray matter 
volume, aiming to find links between distributed structural properties and motor function. Mean MEP classification could be achieved with significant accuracy levels from a widely distributed pattern of voxels composed of regions in frontal, parietal, temporal occipital and cerebellar regions. Similarly, mean $\mathrm{SICI}$ was predicted, with similar accuracy levels, from a widely distributed pattern of voxels which included foci in frontal, parietal, temporal and occipital cortices, as well as the cerebellum. The prediction was specific to mean MEP peak-to-peak amplitudes and SICls. Group differences in the trial-to-trial variation in neither MEPs nor SICls could be classified from patterns of gray matter volume.

Our results reveal that distributed patterns of gray matter volume, extending well beyond M1 allowed for an accurate group classification of MEP and $\mathrm{SICl}$ amplitudes. While MEPs are widely used for probing the physiology of M1, multiple lines of evidence suggest that they do not reflect a simple read-out of neuronal processes occurring within $\mathrm{M1}$, but rather also tap into physiological processes that occur outside of this structure (Bestmann and Krakauer, 2015). This is strongly demonstrated by dual-site stimulation paradigms where a conditioning TMS pulse is delivered to various cortical regions prior to a test stimulus in M1 (Dayan et al., 2013), establishing the functional connectivity of these regions with $\mathrm{M} 1$, while revealing the influence these regions may exert over MEPs (Bestmann and Krakauer, 2015; Liew et al., 2014). Along these lines, several of the regions which were found here as implicated in the classification of MEP and $\mathrm{SICl}$ have been shown to interact with M1 based on dual-site TMS stimulation. For example, connectivity between M1 and ventral premotor (Buch et al., 2010) and dorsal premotor (O'Shea et al., 2007) cortices has been demonstrated, which fits well with the dense inputs between these regions and M1 in the monkey (Dum and Strick, 2005; Hoshi and Tanji, 2007). Similarly, connectivity between the cerebellum and M1 has been demonstrated with dual-site TMS (Daskalakis et al., 2004), consistent with cerebellar -motor cortex loops found in the monkey (Kelly and Strick, 2003). Altogether, using a whole brain analysis and a nonrestricted and unbiased pattern classification method we have not found multivoxel patterns in M1. In summary, our results identify a relationship between distributed multi-voxel patterns of brain volume in extra-motor cortical regions and variability in MEP and SICI. 
In addition to our focus on group classification of differences in corticospinal excitability and inhibition, which we studied by quantifying mean MEPs amplitudes and SICls, we also tested whether there were differences in the trial-to-trial variability subjects display, quantified with the CV statistic. This allowed us to assess the contribution of more transient and unspecific factors to the classification of MEPs and SICls. Contrary to mean MEP and SICl amplitudes, intra-subject variation could not be classified from patterns of gray matter volume. It may thus be that intra-subject variation is indeed induced by more transient and state-dependent factors, which were not controlled for in this study, whereas group differences in mean MEP and $\mathrm{SICI}$ amplitudes were driven by more stable, possibly pre-existing state-independent factors.

Inter- and intraindividual differences in MEP and $\mathrm{SICl}$ amplitudes have been widely reported before, and were ascribed largely to transient, spontaneous and statedependent factors. Variation in MEP measurement, for instance, has been attributed to spontaneous fluctuations in corticospinal and segmental motoneuron excitability (Kiers et al., 1993). Likewise, various transient physiological states such as pre-stimulation muscle activation (Darling et al., 2006), central (Temesi et al., 2014) and more localized (muscle-specific) fatigue (Taylor and Gandevia, 2001), response preparation (Mars et al., 2007) and attention (Rosenkranz and Rothwell, 2004; Thomson et al., 2008) modulate MEPs and SICls. Our results cannot exclude the contribution of transient or spontaneous factors to variability in MEPs and SICls. However, our data suggests that stable, possibly pre-existing non state-dependent neuroanatomical substrates may allow for the classification of group differences in corticospinal excitability and inhibition. Thus, intrinsic (Goetz et al., 2014) and stable properties like brain structure could contribute to the variability in corticospinal excitability and inhibition.

Non-transient factors may also contribute to the variability in MEPs or SICls. For instance, age and sex interact with the trial-to-trial variability of MEP amplitude (Pitcher et al., 2003), but in our current results, none of the classified groups differed in age or in male/female ratios, so the contribution of these factors to the results reported here is unlikely. Similarly, the physical parameters of stimulation may also contribute to the variability in MEPs or SICls, but their contribution to their variability is not trivial. For 
instance, the relationship between mean MEP amplitude and stimulation intensity is not necessarily linear (Darling et al., 2006). Likewise, variability in MEPs does not decrease when more elaborate methods are used to localize M1, such as stereotactic neuronavigation (Jung et al., 2010). The contribution of factors such as stimulation intensity and fluctuations in coil positioning to the degree to which variation in MEPs and SICls relates to brain structure remains to be tested in future research.

As subjects' attention has been found to influence MEPs (Mars et al., 2007) and SICls (Rosenkranz and Rothwell, 2004; Thomson et al., 2008), one possibility that warrants consideration is that the structural differences between subjects who showed higher and lower MEPs and SICls may relate to interindividual differences in attention, which in turn influenced MEP amplitudes and SICls. Regions such as the dorsolateral prefrontal cortex (dIPFC), inferior frontal gyrus, supramarginal gyrus and middle temporal gyrus, found here as implicated in the classification of MEP amplitudes and SICls are considered to be a part of the dorsal and ventral attention networks (Fox et al., 2006; Vossel et al., 2014). Moreover, correlations between regional structural properties of several of the brain regions found here as implicated in the classification of MEP and $\mathrm{SICl}$ with attention or attention-related functions have been reported before (Smolker et al., 2015; Westlye et al., 2010). For instance, significant associations were found between the executive component of attention and cortical thickness in the middle and superior temporal gyri, inferior frontal gyrus and dIPFC, and reduced gray matter volume in dIPFC is associated with better performance in the monitoring and updating of working memory (Smolker et al., 2015), functions which require attentional control (Fougnie, 2008). Future research may explore the relationship between attentionrelated regions and corticospinal excitability.

Although several previous reports found a relationship between SICI and MEP estimates (Roshan et al., 2003; Sanger et al., 2001), a systematic examination revealed that SICls is dependent on the intensity of the test TMS pulse, rather than the size of the test MEP per se (Garry and Thomson, 2009). These results are consistent with our findings that MEP and $\mathrm{SICl}$ amplitudes did not covary among subjects and the regions which contributed to classification of differences along these measures were largely 
independent. Still, because of the lack of covariation in MEPs and SICls among our sample of subjects, the classification analysis for these two measures was based on different subject-groupings. Thus, although our results suggest that differences in MEPs and $\mathrm{SICl}$ amplitudes may possibly originate from variation in largely non-overlapping structural substrates additional data is needed in order to confirm this suggestion.

Our goal here was to establish the feasibility of classifying group differences in MEP and $\mathrm{SICl}$ amplitudes based on multi-voxel patterns of brain volume, aiming to find links between distributed structural properties and motor function. An advantage afforded by this approach is that it enables us to detect subtle and distributed morphological differences between subjects possibly masked by a mass-univariate approach such as voxel-based morphometry (Dayan et al., 2014; Ecker et al., 2010b). However, the supervised learning approach we used here required splitting of the dataset into groups which thus reduced the true variation of the dataset. Links between multivariate representations of brain structure and a more continuous variation in MEP and SICls should be established in future studies.

Conflict of Interest: The authors declare no competing financial interests.

Acknowledgments: This work was supported by the Intramural Research Program of the National Institute of Neurological Disorders and Stroke, National Institutes of Health. Virginia López-Alonso was supported by an FPU fellowship from Ministerio de Educación, Cultura y Deporte, Spain. The study utilized the high-performance computational capabilities of the Biowulf Linux cluster at the National Institutes of Health, Bethesda, Md. (http://biowulf.nih.gov). We thank Ryan Thompson for assistance in the preparation of this manuscript.

Author Contributions: All authors designed the study; V.L.A and S.L.L performed the experiments. E.D and V.L.A analyzed the data. All authors wrote and reviewed the manuscript. 


\section{References}

Bestmann, S., Krakauer, J.W., 2015. The uses and interpretations of the motor-evoked potential for understanding behaviour. Exp. Brain Res. 233, 679-689.

Bishop, C.M., 2006. Pattern recognition and machine learning. springer.

Boser, B.E., Guyon, I.M., Vapnik, V.N., 1992. A training algorithm for optimal margin classifiers, in: Proceedings of the Fifth Annual Workshop on Computational Learning Theory. ACM, pp. 144-152.

Buch, E.R., Mars, R.B., Boorman, E.D., Rushworth, M.F.S., 2010. A network centered on ventral premotor cortex exerts both facilitatory and inhibitory control over primary motor cortex during action reprogramming. J. Neurosci. Off. J. Soc. Neurosci. 30, 1395-1401. doi:10.1523/JNEUROSCI.4882-09.2010

Conde, V., Vollmann, H., Sehm, B., Taubert, M., Villringer, A., Ragert, P., 2012. Cortical thickness in primary sensorimotor cortex influences the effectiveness of paired associative stimulation. Neurolmage 60, 864-870. doi:10.1016/j.neuroimage.2012.01.052

Darling, W.G., Wolf, S.L., Butler, A.J., 2006. Variability of motor potentials evoked by transcranial magnetic stimulation depends on muscle activation. Exp. Brain Res. 174, 376-385. doi:10.1007/s00221-006-0468-9

Daskalakis, Z.J., Paradiso, G.O., Christensen, B.K., Fitzgerald, P.B., Gunraj, C., Chen, R., 2004. Exploring the connectivity between the cerebellum and motor cortex in humans. J. Physiol. 557, 689-700.

Dayan, E., Censor, N., Buch, E.R., Sandrini, M., Cohen, L.G., 2013. Noninvasive brain stimulation: from physiology to network dynamics and back. Nat. Neurosci. 16, 838-844. doi:10.1038/nn.3422

Dayan, E., Hamann, J.M., Averbeck, B.B., Cohen, L.G., 2014. Brain Structural Substrates of Reward Dependence during Behavioral Performance. J. Neurosci. 34, 16433-16441. doi:10.1523/JNEUROSCI.3141-14.2014

Dum, R.P., Strick, P.L., 2005. Frontal lobe inputs to the digit representations of the motor areas on the lateral surface of the hemisphere. J. Neurosci. 25, 13751386.

Ecker, C., Marquand, A., Mourão-Miranda, J., Johnston, P., Daly, E.M., Brammer, M.J., Maltezos, S., Murphy, C.M., Robertson, D., Williams, S.C., others, 2010a.

Describing the brain in autism in five dimensions-magnetic resonance imagingassisted diagnosis of autism spectrum disorder using a multiparameter classification approach. J. Neurosci. 30, 10612-10623.

Ecker, C., Rocha-Rego, V., Johnston, P., Mourao-Miranda, J., Marquand, A., Daly, E.M., Brammer, M.J., Murphy, C., Murphy, D.G., 2010b. Investigating the predictive value of whole-brain structural MR scans in autism: A pattern classification approach. Neurolmage 49, 44-56.

doi:10.1016/j.neuroimage.2009.08.024 
Fougnie, D., 2008. The relationship between attention and working memory. New Res. Short-Term Mem. 1, 45.

Fox, M.D., Corbetta, M., Snyder, A.Z., Vincent, J.L., Raichle, M.E., 2006. Spontaneous neuronal activity distinguishes human dorsal and ventral attention systems. Proc. Natl. Acad. Sci. 103, 10046-10051.

Garry, M.I., Thomson, R.H.S., 2009. The effect of test TMS intensity on short-interval intracortical inhibition in different excitability states. Exp. Brain Res. 193, 267.

Goetz, S.M., Luber, B., Lisanby, S.H., Peterchev, A.V., 2014. A novel model incorporating two variability sources for describing motor evoked potentials. Brain Stimulat. 7, 541-552.

Hallett, M., 2007. Transcranial Magnetic Stimulation: A Primer. Neuron 55, 187-199. doi:10.1016/j.neuron.2007.06.026

He, B.J., Snyder, A.Z., Vincent, J.L., Epstein, A., Shulman, G.L., Corbetta, M., 2007. Breakdown of Functional Connectivity in Frontoparietal Networks Underlies Behavioral Deficits in Spatial Neglect. Neuron 53, 905-918. doi:10.1016/j.neuron.2007.02.013

Hoshi, E., Tanji, J., 2007. Distinctions between dorsal and ventral premotor areas: anatomical connectivity and functional properties. Curr. Opin. Neurobiol. 17, 234-242.

Johansen-Berg, H., Della-Maggiore, V., Behrens, T.E.J., Smith, S.M., Paus, T., 2007. Integrity of white matter in the corpus callosum correlates with bimanual coordination skills. Neurolmage 36, T16-T21. doi:10.1016/j.neuroimage.2007.03.041

Jung, N.H., Delvendahl, I., Kuhnke, N.G., Hauschke, D., Stolle, S., Mall, V., 2010. Navigated transcranial magnetic stimulation does not decrease the variability of motor-evoked potentials. Brain Stimulat. 3, 87-94. doi:10.1016/j.brs.2009.10.003

Kanai, R., Rees, G., 2011. The structural basis of inter-individual differences in human behaviour and cognition. Nat. Rev. Neurosci. 12, 231-242. doi:10.1038/nrn3000

Kelly, R.M., Strick, P.L., 2003. Cerebellar loops with motor cortex and prefrontal cortex of a nonhuman primate. J. Neurosci. 23, 8432-8444.

Kiers, L., Cros, D., Chiappa, K.H., Fang, J., 1993. Variability of motor potentials evoked by transcranial magnetic stimulation. Electroencephalogr. Clin. Neurophysiol. 89, 415-423.

Kujirai, T., Caramia, M.D., Rothwell, J.C., Day, B.L., Thompson, P.D., Ferbert, A., Wroe, S., Asselman, P., Marsden, C.D., 1993. Corticocortical inhibition in human motor cortex. J. Physiol. 471, 501-519.

Liew, S.-L., Santarnecchi, E., Buch, E.R., Cohen, L.G., 2014. Non-invasive brain stimulation in neurorehabilitation: local and distant effects for motor recovery. Front. Hum. Neurosci. 8. doi:10.3389/fnhum.2014.00378

Marquand, A., Howard, M., Brammer, M., Chu, C., Coen, S., Mourão-Miranda, J., 2010. Quantitative prediction of subjective pain intensity from whole-brain $\mathrm{fMRI}$ data using Gaussian processes. Neuroimage 49, 2178-2189.

Mars, R.B., Bestmann, S., Rothwell, J.C., Haggard, P., 2007. Effects of motor preparation and spatial attention on corticospinal excitability in a delayedresponse paradigm. Exp. Brain Res. 182, 125-129. 
Matsumoto, R., Nair, D.R., LaPresto, E., Bingaman, W., Shibasaki, H., Luders, H.O., 2006. Functional connectivity in human cortical motor system: a cortico-cortical evoked potential study. Brain 130, 181-197. doi:10.1093/brain/awl257

Mourão-Miranda, J., Bokde, A.L., Born, C., Hampel, H., Stetter, M., 2005. Classifying brain states and determining the discriminating activation patterns: support vector machine on functional MRI data. Neurolmage 28, 980-995.

Oldfield, R.C., 1971. The assessment and analysis of handedness: the Edinburgh inventory. Neuropsychologia 9, 97-113.

O'Shea, J., Sebastian, C., Boorman, E.D., Johansen-Berg, H., Rushworth, M.F.S., 2007. Functional specificity of human premotor-motor cortical interactions during action selection. Eur. J. Neurosci. 26, 2085-2095. doi:10.1111/j.14609568.2007.05795.x

Picard, N., Strick, P.L., 1996. Motor areas of the medial wall: a review of their location and functional activation. Cereb. Cortex 6, 342-353.

Pitcher, J.B., Ogston, K.M., Miles, T.S., 2003. Age and sex differences in human motor cortex input-output characteristics. J. Physiol. 546, 605-613.

doi:10.1113/jphysiol.2002.029454

Rosenkranz, K., Rothwell, J.C., 2004. The effect of sensory input and attention on the sensorimotor organization of the hand area of the human motor cortex: Muscle vibration and sensorimotor organization. J. Physiol. 561, 307-320. doi:10.1113/jphysiol.2004.069328

Roshan, L., Paradiso, G.O., Chen, R., 2003. Two phases of short-interval intracortical inhibition. Exp. Brain Res. 151, 330-337.

Rothwell, J.C., 1997. Techniques and mechanisms of action of transcranial stimulation of the human motor cortex. J. Neurosci. Methods 74, 113-122.

Rothwell, J.C., Day, B.L., Thompson, P.D., Kujirai, T., 2009. Short latency intracortical inhibition: one of the most popular tools in human motor neurophysiology: Perspectives. J. Physiol. 587, 11-12. doi:10.1113/jphysiol.2008.162461

Rothwell, J.C., Hallett, M., Berardelli, A., Eisen, A., Rossini, P., Paulus, W., 1999. Magnetic stimulation: motor evoked potentials. Electroencephalogr Clin Neurophysiol Suppl 52, 97-103.

Sakai, K., Ugawa, Y., Terao, Y., Hanajima, R., Furubayashi, T., Kanazawa, I., 1997. Preferential activation of different I waves by transcranial magnetic stimulation with a figure-of-eight-shaped coil. Exp. Brain Res. 113, 24-32.

Sanger, T.D., Garg, R.R., Chen, R., 2001. Interactions between two different inhibitory systems in the human motor cortex. J. Physiol. 530, 307-317.

Smolker, H.R., Depue, B.E., Reineberg, A.E., Orr, J.M., Banich, M.T., 2015. Individual differences in regional prefrontal gray matter morphometry and fractional anisotropy are associated with different constructs of executive function. Brain Struct. Funct. 220, 1291-1306.

Taylor, J.L., Gandevia, S.C., 2001. Transcranial magnetic stimulation and human muscle fatigue. Muscle Nerve 24, 18-29.

Temesi, J., Rupp, T., Martin, V., Arnal, P.J., FéAsson, L., Verges, S., Millet, G.Y., 2014. Central Fatigue Assessed by Transcranial Magnetic Stimulation in Ultratrail Running: Med. Sci. Sports Exerc. 46, 1166-1175. doi:10.1249/MSS.0000000000000207 
Thomson, R.H.S., Garry, M.I., Summers, J.J., 2008. Attentional influences on shortinterval intracortical inhibition. Clin. Neurophysiol. 119, 52-62. doi:10.1016/j.clinph.2007.09.060

van Gaal, S., Scholte, H.S., Lamme, V.A.F., Fahrenfort, J.J., Ridderinkhof, K.R., 2011. Pre-SMA Gray-matter Density Predicts Individual Differences in Action Selection in the Face of Conscious and Unconscious Response Conflict. J. Cogn. Neurosci. 23, 382-390. doi:10.1162/jocn.2010.21444

Vemuri, P., Gunter, J.L., Senjem, M.L., Whitwell, J.L., Kantarci, K., Knopman, D.S., Boeve, B.F., Petersen, R.C., Jack, C.R., 2008. Alzheimer's disease diagnosis in individual subjects using structural MR images: Validation studies. Neurolmage 39, 1186-1197. doi:10.1016/j.neuroimage.2007.09.073

Vossel, S., Geng, J.J., Fink, G.R., 2014. Dorsal and ventral attention systems: distinct neural circuits but collaborative roles. The Neuroscientist 20, 150-159.

Westlye, L.T., Grydeland, H. akon, Walhovd, K.B., Fjell, A.M., 2010. Associations between regional cortical thickness and attentional networks as measured by the attention network test. Cereb. Cortex 21, 345-356. 


\section{Figures}

A.

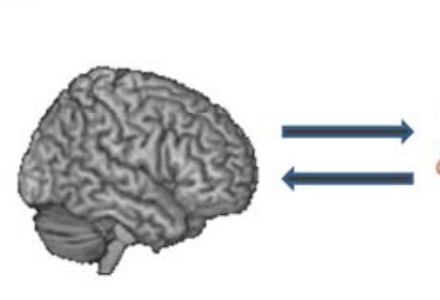

Scanning Session

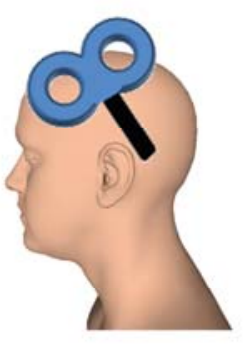

TMS Session

c. Training Dataset
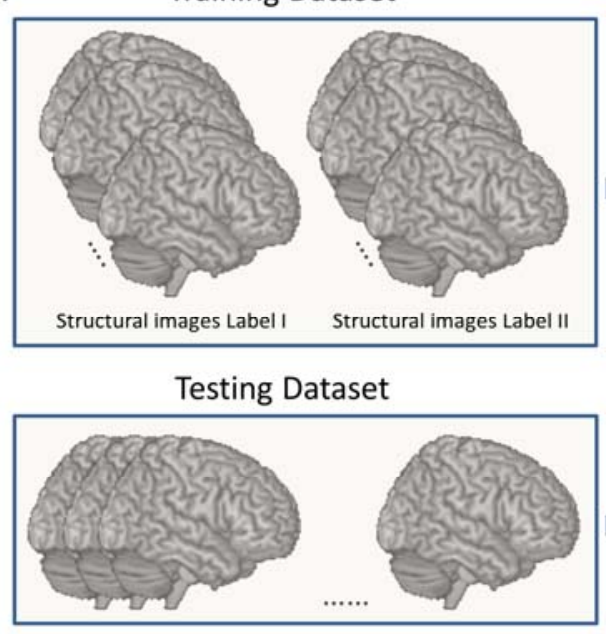

B.
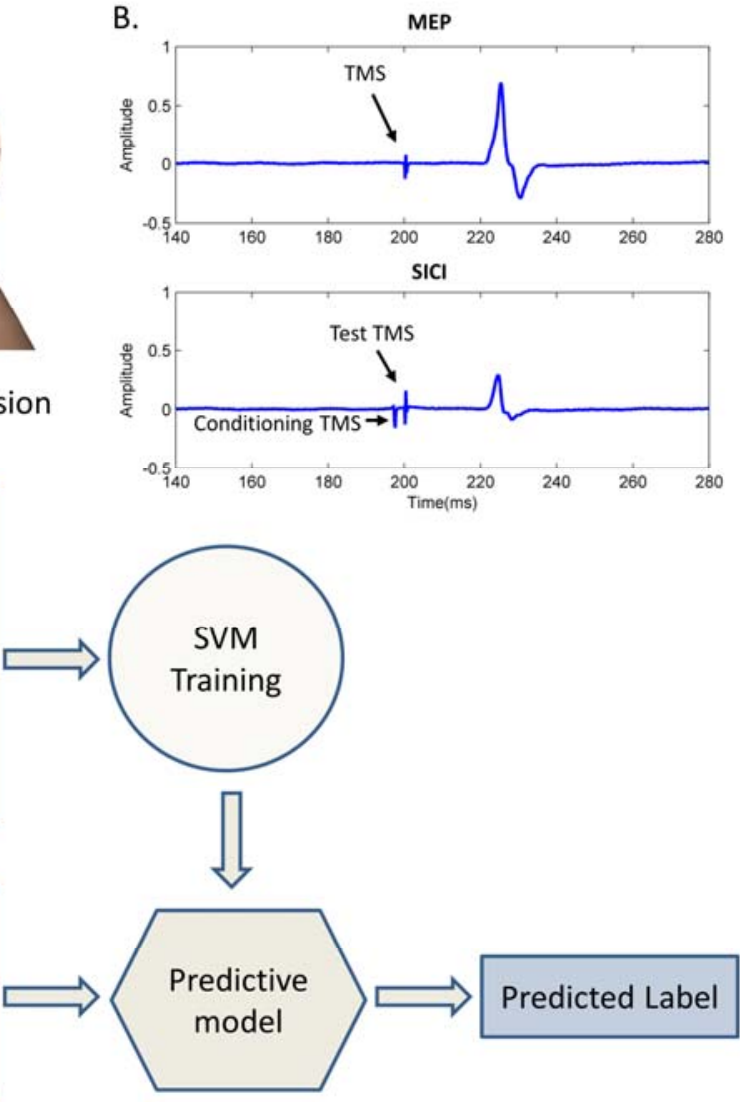

Predictive model

Predicted Label

Fig. 1. Design and analysis. (A) All subjects underwent a TMS session, where single and paired pulses were used to measures MEPs and SICls and an imaging session where high resolution T1 weighted images were acquired, (B) MEPs were recorded from the FDI muscle following single TMS pulses delivered over primary motor cortex at $120 \%$ RMT. SICls were recorded with a subthreshold conditioning stimulus at $80 \%$ RMT followed by a test stimulus at $120 \%$ RMT. (C) Pattern classification analysis was composed of two phases. In the training phase, a kernel SVM classifier was trained to distinguish between sets of anatomical images with known labels. A hyperplane that separates the training images according to their known labels was defined. Then, during the test phase, the performance of the classifier is tested on unlabeled images testing if the predictive model built during training can successfully classify the images. Fig $1 \mathrm{~A}$ was drawn based on a human head model from: http://www.ir-Itd.net/. Used by Creative Commons license. 

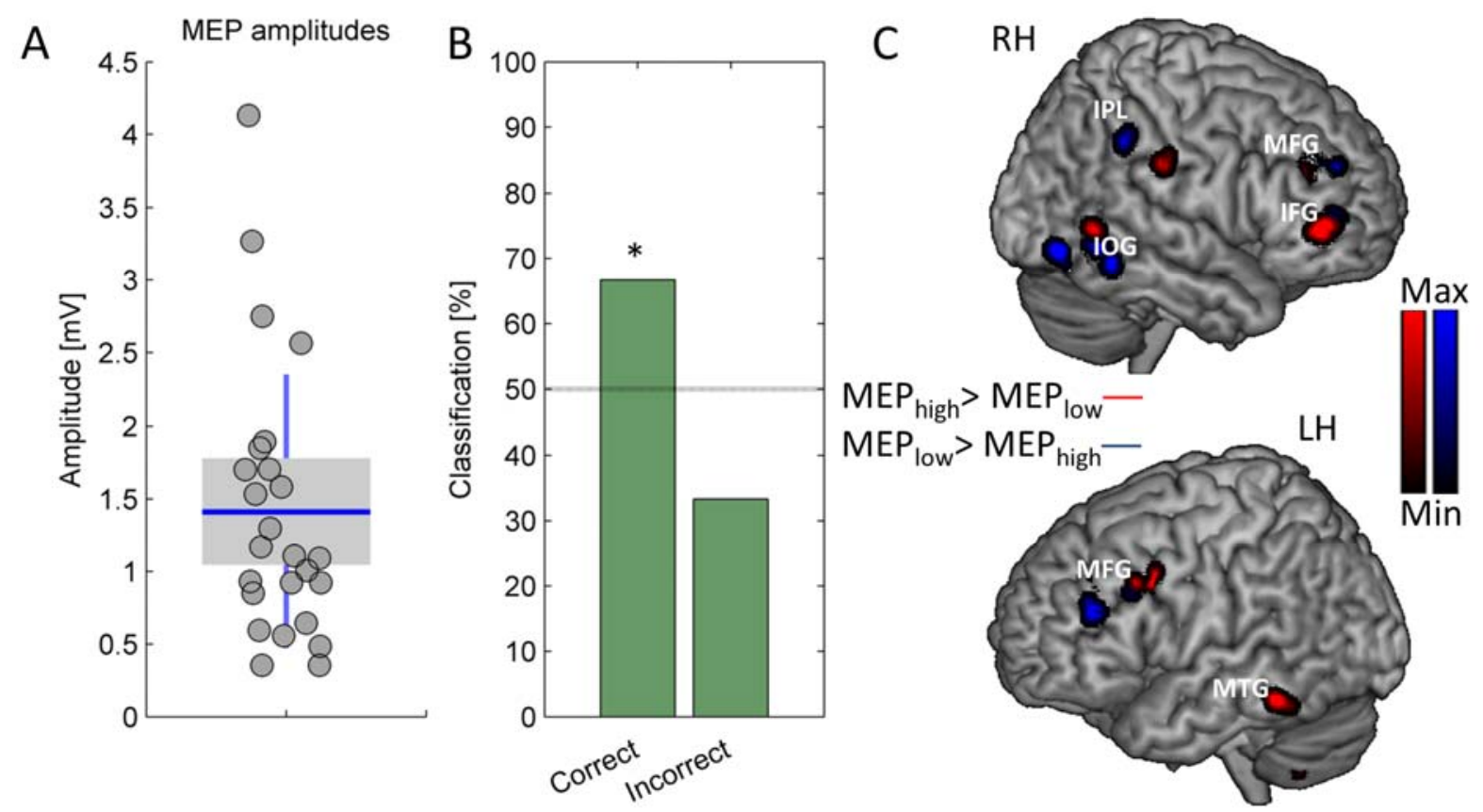

Fig. 2. Pattern classification of MEP amplitudes. (A) Mean peak-to-peak MEPs differed by up to $168.4 \%$ among subjects. (B) Pattern classification of MEP amplitudes. Overall classification accuracy was significant at $p<0.05$, (random permutation test) (C) Discrimination maps depicting the weight of the voxels which contributed the most to the discrimination among the subjects who displayed low and high MEP amplitudes. Shown are regions where $M E P_{\text {low }}>M E P_{\text {high }}$ (in blue) and where $M E P_{\text {high }}>M E P_{\text {low }}$ (in red). LH, left hemisphere; $\mathrm{RH}$, right hemisphere. IFG, inferior frontal gyrus; IOG, inferior occipital gyrus; IPL, inferior parietal lobule; MFG, middle frontal gyrus; MTG, middle temporal gyrus. 


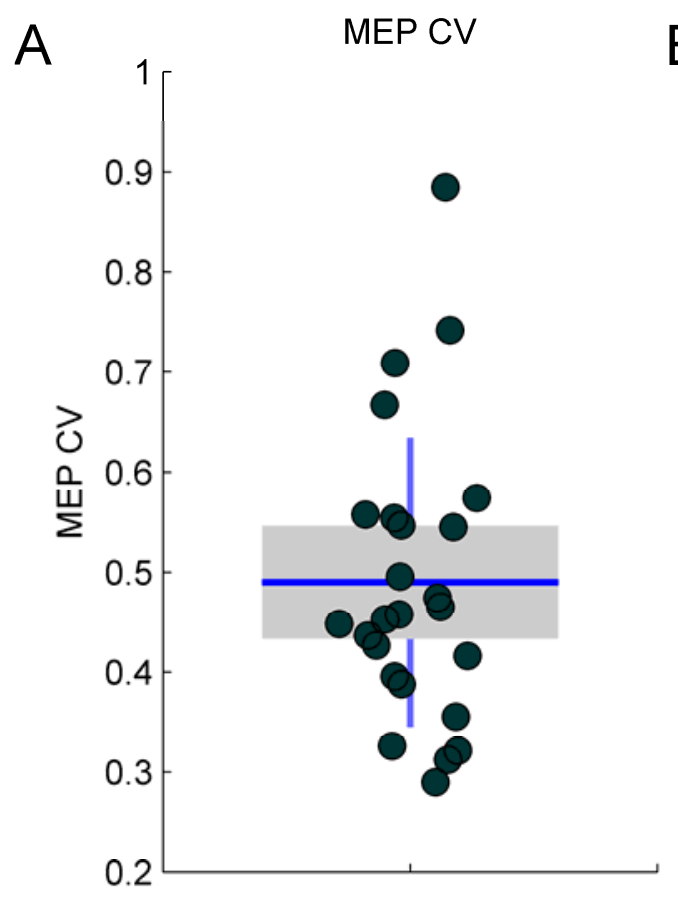

B

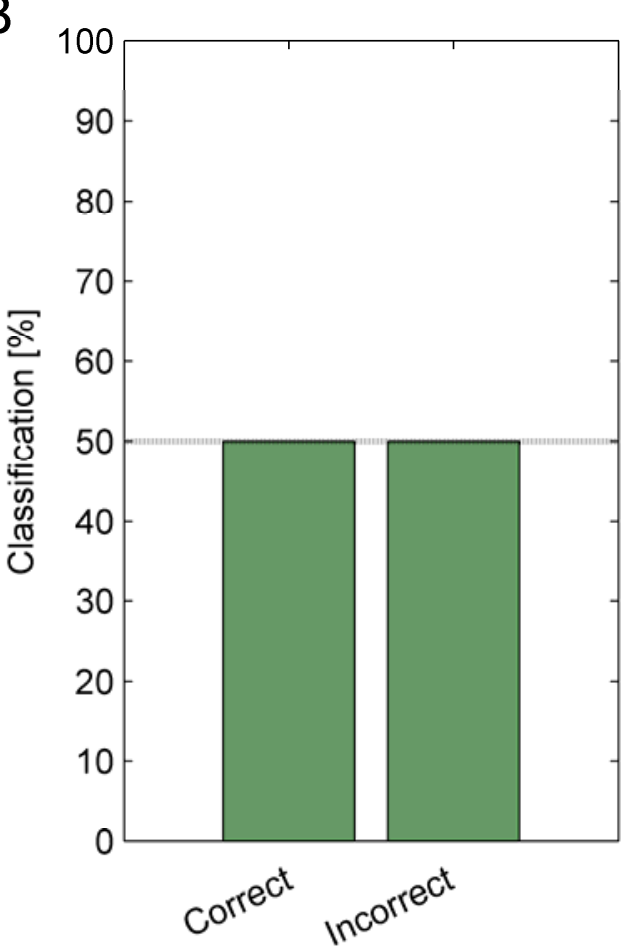

Fig. 3. Pattern classification of MEP trial-to-trial variation. (A) Trial-to-trial variability in MEP amplitudes, quantified with CV statistic, differed among subjects by up to $101.3 \%$ (B) Overall classification accuracy was at chance levels (50\%) 

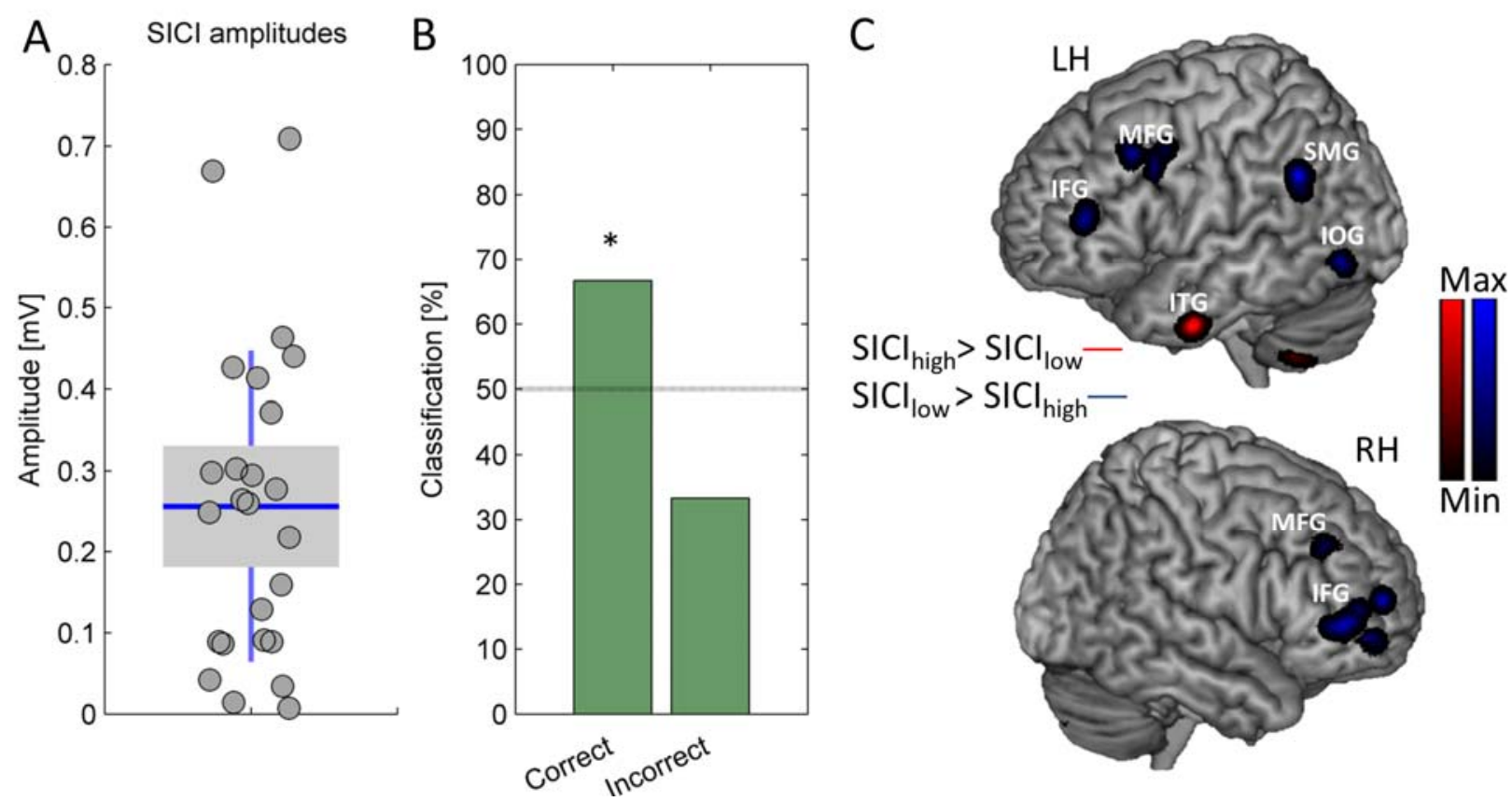

Fig. 4. Pattern classification of SICI amplitudes. (A) Mean peak-to-peak SICls differed by up to $195.83 \%$ among subjects. (B) Overall classification accuracy was significant at $p<0.05$, (random permutation test) $C$. Discrimination maps depicting the weight of the voxels which contributed the most to the discrimination among the subjects who displayed low and high MEP amplitudes. Shown are regions where $\mathrm{SICl}_{\text {ow }}>\mathrm{SICl}_{\text {high }}$ (in blue) and where $\mathrm{SICl}_{\text {high }}>\mathrm{SICl}_{\text {low }}$ (in red). $\mathrm{LH}$, left hemisphere; $\mathrm{RH}$, right hemisphere. IFG, inferior frontal gyrus; IOG, inferior occipital gyrus; ITG, inferior temporal; gyrus MFG, middle frontal gyrus; SMP, supramaginal gyrus. 

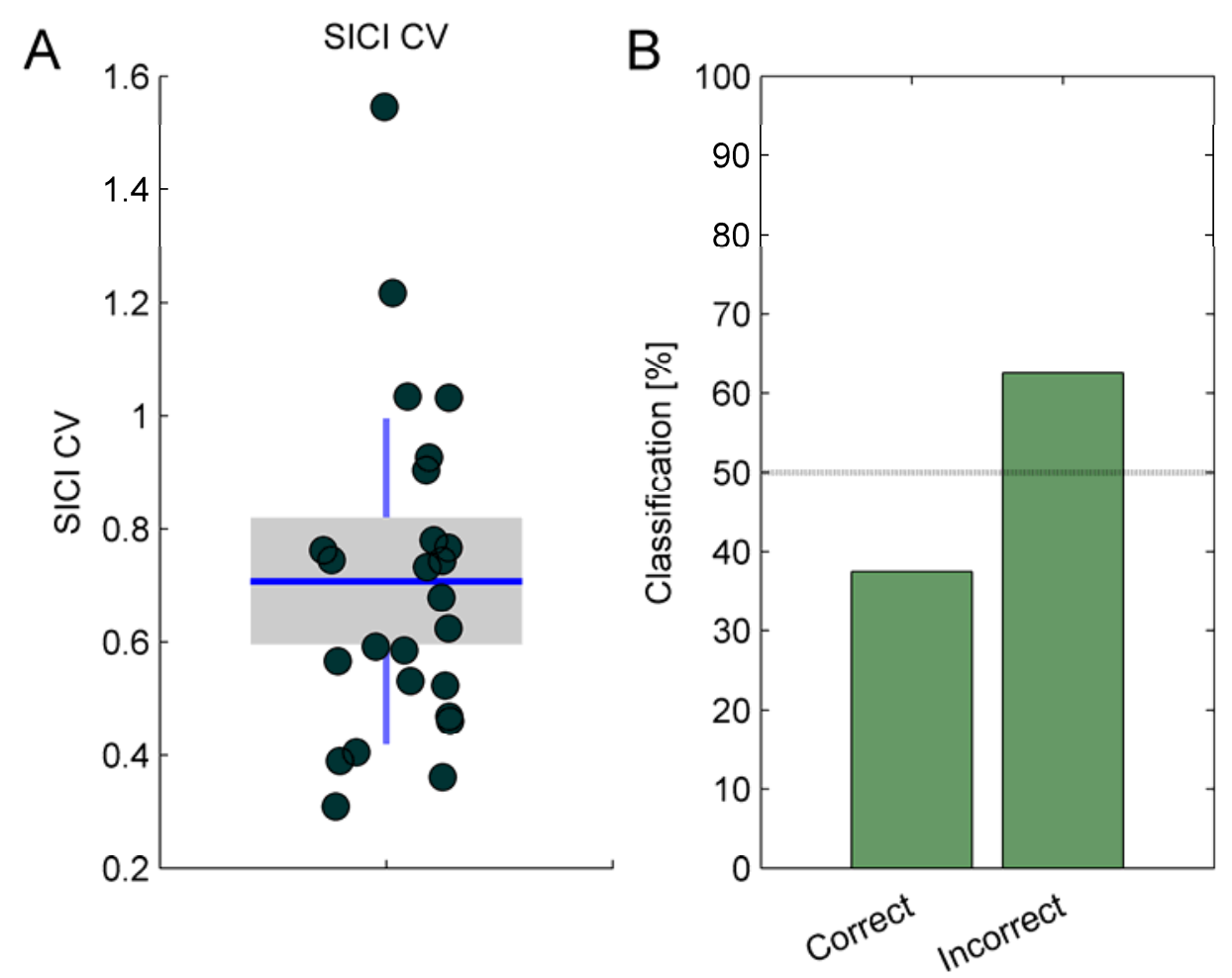

Fig. 5. Pattern classification of $\mathrm{SICl}$ trial-to-trial variation. (A) Trial-to-trial variability in $\mathrm{SICl}$ amplitudes, quantified with CV statistic, differed among subjects by up to $133.3 \%$ (B) Overall classification accuracy was worse than chance levels. 


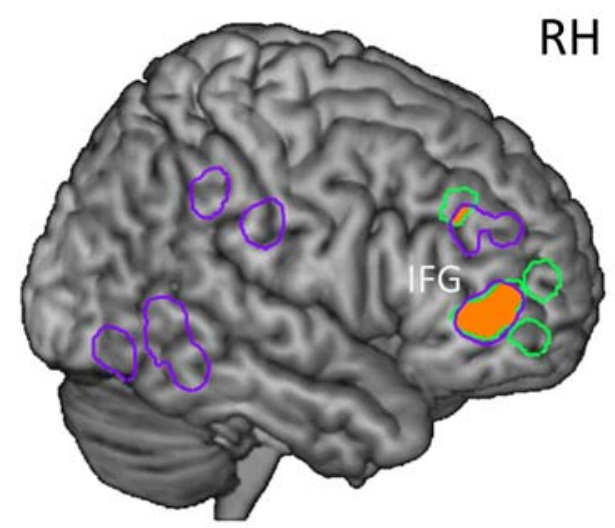

MEP Classification SICl classification

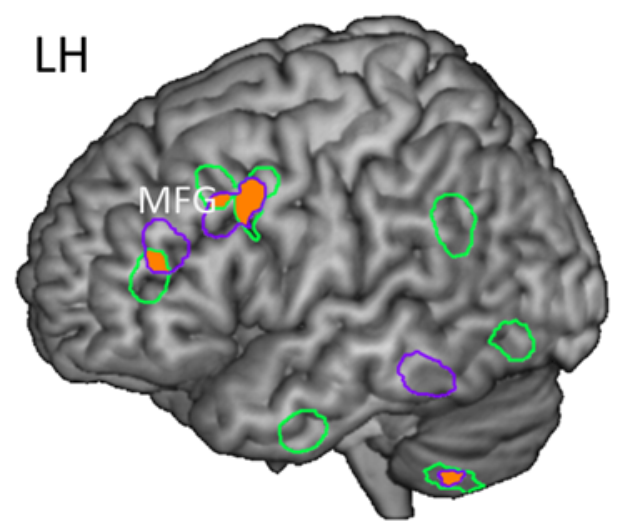

Fig. 6. Similarities in the classification of $\mathrm{MEP}$ and $\mathrm{SICl}$ amplitudes. Overlaying the discrimination maps identified in the classification of each of these measures (depicted in purple and green contours) revealed that apart from a partial overlap in bilateral frontal cortex and right cerebellum (overlap in orange) the maps were mostly nonoverlapping. IFG, inferior frontal gyrus; MFG, middle frontal gyrus. 


\section{Tables}

\section{Table 1}

Brain regions that contributed the most to the discrimination among the subjects who displayed low and high MEP amplitudes. X,Y, Z coordinates (in MNI space) are displayed.

\begin{tabular}{|c|c|c|c|c|}
\hline Region & side & $\mathbf{X}$ & $\mathbf{Y}$ & $\mathbf{Z}$ \\
\hline \multicolumn{5}{|l|}{$M E P_{\text {low }}>M E P_{\text {high }}$} \\
\hline Inferior Occipital Gyrus & $\mathrm{R}$ & 40.5 & -69 & -10.5 \\
\hline Middle Frontal Gyrus & $\mathrm{R}$ & 22 & 37 & 29 \\
\hline Middle Frontal Gyrus & $\mathrm{L}$ & -37.5 & 37.5 & 22.5 \\
\hline Inferior Parietal Lobule & $\mathrm{R}$ & 52.5 & -39 & 40.5 \\
\hline \multicolumn{5}{|l|}{$M E P_{\text {high }}>M E P_{\text {low }}$} \\
\hline Posterior Cerebellum & $\mathrm{L}$ & -31.5 & -52.5 & -49.5 \\
\hline Middle Temporal Gyrus & L & -55.5 & -40.5 & -15 \\
\hline Middle Temporal Gyrus & $\mathrm{R}$ & 56 & -52.5 & 3 \\
\hline Inferior Frontal Gyrus & $\mathrm{R}$ & 37.5 & 45 & 3 \\
\hline Middle Frontal Gyrus & $\mathrm{R}$ & 27 & 37 & 25 \\
\hline Middle Frontal Gyrus & L & -37.5 & 10.5 & 36 \\
\hline Postcentral Gyri & $\mathrm{R}$ & 58.5 & -22.5 & 33 \\
\hline
\end{tabular}




\section{Table 2}

Brain regions that contributed the most to the discrimination among the subjects who displayed low and high SICl amplitudes. X,Y, Z coordinates (in MNI space) are displayed.

\begin{tabular}{|c|c|c|c|c|}
\hline Region & side & $\mathbf{X}$ & $\mathbf{Y}$ & $\mathbf{Z}$ \\
\hline \multicolumn{5}{|l|}{$S I C I_{\text {low }}>S I C I_{\text {high }}$} \\
\hline Inferior Occipital Gyrus & L & -39 & -70.5 & -7.5 \\
\hline Inferior Frontal Gyrus & $\mathrm{R}$ & 39 & 45 & 3 \\
\hline Inferior Frontal Gyrus & L & -37 & 42 & 13 \\
\hline Supramarginal Gyrus & L & -51 & -51 & 31.5 \\
\hline Middle Frontal Gyrus & $\mathrm{R}$ & 25 & 34 & 35 \\
\hline Middle Frontal Gyrus & L & -34 & 19.5 & 40.5 \\
\hline \multicolumn{5}{|l|}{$S I C I_{\text {high }}>S I C I_{\text {low }}$} \\
\hline \multirow[t]{2}{*}{ Posterior Cerebellum } & L & -31.5 & -51 & -49.5 \\
\hline & $\mathrm{R}$ & 12 & -84 & -37.5 \\
\hline Inferior Temporal Gyrus & $\mathrm{L}$ & -46.5 & -4.5 & -33 \\
\hline
\end{tabular}

